Open Access

\title{
Erratum to: Elafin is downregulated during breast and ovarian tumorigenesis but its residual expression predicts recurrence
}

Joseph A Caruso ${ }^{1,5,7}$, Cansu Karakas 1,2, Jing Zhang², Min Yi ${ }^{3}$, Constance Albarracin², Aysegul Sahin², Melissa Bondy ${ }^{4}$, Jinsong Liu ${ }^{2,5}$, Kelly K. Hunt ${ }^{3}$ and Khandan Keyomarsi ${ }^{1,5,6^{*}}$

\section{Erratum}

During the editorial process preceding the publication of this article an error was introduced in the heading of "Table 1 Univariate and multivariate analysis of elafinpositive cells in breast cancer patients." This error was only noticed after publication of the final version. Specifically, both data-containing columns are labeled "Elafin Negative." The data-containing column on the right should in fact be labeled "Elafin Positive $(\mathrm{n}=482)$." The corrected version can be found here (Table 1). Table 1 has also been updated in the original article to reflect the correct labeling of the columns.

\footnotetext{
Author details

'Department of Experimental Radiation Oncology, University of Texas at MD Anderson Cancer Center, 1515 Holcombe Blvd., Houston, TX 77030, USA. ${ }^{2}$ Department of Pathology, University of Texas at MD Anderson Cancer Center, 1515 Holcombe Blvd., Houston, TX 77030, USA. ${ }^{3}$ Department of Surgical Oncology, University of Texas at MD Anderson Cancer Center, 1515 Holcombe Blvd., Houston, TX 77030, USA. ${ }^{4}$ Baylor College of Medicine, Dan L. Duncan Cancer Center, 1 Baylor Plaza, Houston, Texas 77030, USA. ${ }^{5}$ The University of Texas Graduate School of Biomedical Sciences, 6767 Bertner Ave, Houston, Texas 77030, USA. ${ }^{6}$ Department of Experimental Oncology, UT, M.D. Anderson Cancer Center, 1515 Holcombe Blvd., Unit 066, Houston, Texas 77030-4009, USA. ${ }^{7}$ Department of Pathology, UC San Francisco, 513 Parnassus Avenue, San Francisco, CA 94143-0511, USA.
}

Received: 15 April 2015 Accepted: 15 April 2015

Published online: 25 June 2015

\section{References}

1. Caruso JA, Karakas C, Zhang J, Yi M, Albarracin C, Sahin A, et al. Elafin is downregulated during breast and ovarian tumorigenesis but its residual expression predicts recurrence. Breast Canc Res. 2014;16:3417.

\footnotetext{
* Correspondence: kkeyomar@mdanderson.org

'Department of Experimental Radiation Oncology, University of Texas at MD Anderson Cancer Center, 1515 Holcombe Blvd., Houston, TX 77030, USA ${ }^{5}$ The University of Texas Graduate School of Biomedical Sciences, 6767 Bertner Ave, Houston, Texas 77030, USA

Full list of author information is available at the end of the article
}

\section{Submit your next manuscript to BioMed Central and take full advantage of:}

- Convenient online submission

- Thorough peer review

- No space constraints or color figure charges

- Immediate publication on acceptance

- Inclusion in PubMed, CAS, Scopus and Google Scholar

- Research which is freely available for redistribution 
Table 1 Univariate and multivariate analysis of elafin-positive cells in breast cancer patients

\begin{tabular}{|c|c|c|c|}
\hline \multicolumn{4}{|c|}{ A. Univariate analysis, breast cancer $(n=793)$} \\
\hline Factors & $\begin{array}{l}\text { Elafin negative } \\
(\mathrm{n}=311)\end{array}$ & $\begin{array}{l}\text { Elafin positive } \\
(n=482)\end{array}$ & $p$-value \\
\hline Age of diagnosis, year & & & 0.02 \\
\hline Mean & 55.5 & 53.5 & \\
\hline Median (range) & $55(26-86)$ & $52(25-87)$ & \\
\hline Stage & & & $<0.0001$ \\
\hline 1 & $120(38.7)$ & $115(24.0)$ & \\
\hline$\| A$ & $128(41.3)$ & $241(50.2)$ & \\
\hline$\| \mathrm{B}$ & $62(20.0)$ & $124(25.8)$ & \\
\hline Unknown & 1 & 2 & \\
\hline ER & & & $<0.0001$ \\
\hline Positive & $266(85.5)$ & $280(58.3)$ & \\
\hline Negative & $45(14.5)$ & $200(41.7)$ & \\
\hline Unknown & 0 & 2 & \\
\hline$P R$ & & & $<0.0001$ \\
\hline Positive & $218(70.1)$ & $236(49.2)$ & \\
\hline Negative & $93(29.9)$ & $244(50.8)$ & \\
\hline Unknown & 0 & 2 & \\
\hline HER-2 & & & 0.013 \\
\hline Positive & $42(13.5)$ & $98(20.4)$ & \\
\hline Negative & $269(86.5)$ & 383 (79.6) & \\
\hline Unknown & 0 & 1 & \\
\hline Grade & & & $<0.0001$ \\
\hline 1 & $41(14.2)$ & $31(6.8)$ & \\
\hline$\|$ & 186 (64.6) & 197 (43.5) & \\
\hline III & $61(21.2)$ & $225(49.7)$ & \\
\hline Unknown & 23 & 29 & \\
\hline Tumor subtype & & & $<0.0001$ \\
\hline Luminal A & $185(61.9)$ & $158(33.1)$ & \\
\hline Luminal B & $45(15.1)$ & 94 (19.8) & \\
\hline Her2 positive & $42(14.0)$ & 98 (20.7) & \\
\hline Triple negative & $27(9.0)$ & $125(26.4)$ & \\
\hline Unknown & 12 & 7 & \\
\hline Recurrence & & & $<0.0001$ \\
\hline No & $250(80.9)$ & $298(62.3)$ & \\
\hline Yes & $59(19.1)$ & $180(37.7)$ & \\
\hline Unknown & 2 & 4 & \\
\hline
\end{tabular}

Table 1 Univariate and multivariate analysis of elafin-positive cells in breast cancer patients (Continued)

B. Multivariate Cox proportional hazards analysis of clinicopathologic variables' influence on breast cancer RFS in whole cohort $(n=793)$

\begin{tabular}{cllll} 
Factor & HR & Se & P & $95 \% \mathrm{Cl}$ \\
Stage & & & & \\
I & referent & & & \\
IIA & 1.67 & 0.31 & 0.005 & $1.17-2.39$ \\
IIB & 2.41 & 0.47 & $<0.0001$ & $1.65-3.52$ \\
Age & 0.98 & 0.01 & $<0.0001$ & $0.97-0.99$ \\
Elafin Positive & 1.94 & 0.3 & $<0.0001$ & $1.44-2.62$ \\
\hline
\end{tabular}

